\title{
N87-20075:
}

\section{Radiation of Plasma Waves by a Conducting Body Moving Through a Magnetized Plasma ${ }^{\dagger}$}

by

\author{
Alan Barnett and Stanislaw Olbert \\ Massachusetts Institute of Technology
}

\begin{abstract}
An understanding of the interaction between a moving conductor and a plasma is very important for the design of large structures in space, such as a space station or the thered satellite system. It is well known that very large conducting objects which move slowly across magnetic field lines radiate low frequency (Alfvén) waves. In this paper we formulate the problem in such a way that the radiation in all frequency bands can be computed. We then quote results of detailed calculations for spheres and cylinders of various sizes in the cold plasma approximation. In general, we find that in a plasma for which $\omega_{p}^{2} \gg>\omega_{e}^{2}$ and $V^{2} \ll c_{A}^{2} \ll<c^{2}$, there is radiation in three frequency bands: $\omega<\Omega_{i}, \omega_{l h}<\omega<\Omega_{e}, \omega_{p}<\omega<\omega_{u h}$, where $V$ is the speed of the body, $c_{A}$ is the Alfven speed, $c$ is the speed of light in vacuum, $\omega$ is the frequency of the radiation, and $\Omega_{i}, \omega_{l h}, \Omega_{e}, \omega_{p}$, and $\omega_{u h}$ are the ion cyclotron, lower hybrid, electron cyclotron, plasma, and upper hybrid frequencies, respectively.
\end{abstract}

\section{Statement of the problem}

We consider the problem of a conductor moving nonrelativistically through a magnetized plasma with velocity $\mathbf{V}=V \mathbf{e}_{\boldsymbol{x}}$. We call the rest frame of the plasma $\Sigma$ and the rest frame of the conductor $\Sigma^{\prime}$. We assume that in $\Sigma$ far from the conductor the plasma is uniform and isotropic and has a magnetic field

$$
\mathbf{B}_{0}=B_{0} \mathbf{e}_{z}
$$

and an electric field

$$
\mathrm{E}_{0}=0
$$

In $\Sigma^{\prime}$ the fields far from the body are given by

$$
\mathbf{B}_{0}^{\prime}=B_{0} \mathbf{e}_{2}
$$

and

$$
\mathbf{E}^{\prime}=\frac{\mathbf{V} \times \mathbf{B}_{0}}{c}=-\frac{V B_{0}}{c} \mathbf{e}_{y}
$$

Inside the conductor, the electric field $\mathbf{E}^{\prime}$ and the conduction current density $\mathbf{J}_{c}^{\prime}$ are related by

$\dagger$ This paper is a shortened version of the paper of the same name that appeared in the Journal of Geophysical Research, Vol 91, No A9, pages 10, 117-10, 135, September 1, 1986. 


$$
\mathbf{J}_{c}^{\prime}=\sigma \mathbf{E}^{\prime}
$$

In $\Sigma, \mathrm{Eq}(5)$ takes the form

$$
\mathbf{E}=-\frac{\mathbf{V} \times \mathbf{B}_{0}}{c}+\frac{\mathbf{J}_{c}}{\sigma}
$$

We consider here the special case where $\mathbf{V}$ is perpendicular to $\mathbf{B}_{0}$. Our formulation can be easily modified to include the general case. The coordinate system that we use is shown in Fig. 1.

A conductor placed in an electric field in vacuum becomes polarized so as to shield out the external field. The equipotential surfaces for a prolate spheroid embedded in an external electric field directed along its axis is shown in Fig. 2. The field has azimuthal symmetry about the axis of the spheroid.

If the conductor is embedded in a plasma, the above solution cannot apply, since the component of the electric field parallel to the magnetic field will drive currents in the plasma. In particular, for an object is much longer in the direction of the external field than in the direction perpendicular to the field, such as the prolate spheroid shown in Fig. 2, the fields near the tips are much stronger than the field at infinity.

Let $L$ be a characteristic size of the body and $c_{A}$ the Alfvén speed. If the body is large enough and is moving slowly enough $\left(V \ll c_{A}\right.$ and $\left.V / L \Omega_{i} \ll 1\right)$, then the MHD approximation is applicable. The MHD model of the interaction between a moving conductor and a magnetized is based on the work of Drell, Foley and Ruderman (1965), who used it to explain the anomolously fast decay of the orbit of the Echo I weather satellite. The model did not gain wide acceptance until it was confirmed by in situ observations by Voyager I of the Alfvén wing generated by the jovian satellite Io. Further theoretical work was done by Neubauer, and good agreement to the model was obtained in analysis of the magnetic field data by Acuna et al (1981) and of the plasma data by Barnett (1986). In this limit, the conductor radiates Alfvén waves. Viewed in $\Sigma^{\prime}$, there is a standing wave pattern consisting of two Alfvern "wings" attached to the body. The wings have a cross-section determined by the shape of the body, and they extend in the direction of the Alfvén characteristics, $V_{A}^{ \pm}$, defined by

$$
\mathbf{V}_{A}^{ \pm}=\mathbf{V} \pm \frac{\mathbf{B}_{0}}{\sqrt{4 \pi \rho}}
$$

where $\rho$ is the plasma mass density. A side view of the Alfvén wing for a perfectly conducting sphere is shown in Fig. 3. The electric field $\mathbf{E}^{\prime}$ is zero inside the Alfvén wings, while outside them the electric field resembles the field that surrounds a conducting infinite cylinder emersed in a uniform electric field perpendicular to its axis. The external electric field is shielded out by charges on the surface of the wing. In addition, electric current flows along $\mathbf{B}$ on the surface of the wing. The current flows toward the sphere on the side of the wing that is negatively charged, crosses the magnetic field through the sphere, and flows away from the sphere on the side of the wing that is positively charged. A front view of the Alfven wing, showing schematically the charge and current density, is shown in Fig. 4. The electric field in a plane perpendicular to $\mathbf{B}$ through the center of the sphere is shown in Fig. 5. The plasma bulk velocity $\mathrm{V}^{\prime}$ resembles the flow of an incompressible fluid around and infinite cylinder. 
The streamlines in a plane perpendicular to $\mathbf{B}$ are shown in Fig. 6. An interesting property of the Alfven wing system is that a three dimensional problem has effectively become a two dimensional one. Instead of flowing around a spherical obstacle, the plasma must flow around a cylindrical one.

Also shown in Figs. 3 and 4 is the direction of the Poynting vector S. Viewed in $\Sigma$, the conductor loses energy due to radiation. This radiation is analogous to classical Cherenkov radiation, which occurs whenever a particle moves through a dielectric faster than the phase speed of light in the dielectric. In the case of the Alfven wing, the conductor is moving accross the magnetic field faster than the Alfven waves can propagate in that direction (the phase velocity of Alfvén waves perpendicular to $\mathbf{B}$ is zero). If the velocity of the conductor were greater than the Alfvén speed, a shock would form instead of the Alfvén wing.

The question remains, are the MHD waves predicted by Drell et al the only waves generated by a conductor moving through a plasma, or are there waves of higher frequencies? If other waves are possible, how does one compute the amount of energy radiated?

\section{Mathematical Formulation}

We now proceed to the formulation of the problem without the MHD frequency constraint. We solve the problem using Fourier transforms. Our method incorporates the boundary conditions in an integral equation. We start by writing

$$
\nabla \times(\nabla \times \mathbf{E})+\frac{1}{c^{2}} \frac{\partial^{2} \mathbf{E}}{\partial t^{2}}=-\frac{4 \pi}{c^{2}} \frac{\partial \mathbf{J}}{\partial t}
$$

We now write $\mathbf{J}$ as a sum of two terms

$$
\mathbf{J}=\mathbf{J}_{p}(1-H)+\mathbf{J}_{c} H
$$

or

where

$$
\mathbf{J}=\mathbf{J}_{c}-\mathbf{J}_{s}
$$

(where

and

$$
\mathbf{J}_{s}(\mathbf{x}, t)=\left(\mathbf{J}_{c}(\mathbf{x}, t)-\mathbf{J}_{p}(\mathbf{x}, t)\right) H(\mathbf{x}, t)
$$

$$
H(\mathbf{x}, t)= \begin{cases}1 & \text { inside the conductor } \\ 0 & \text { elsewhere }\end{cases}
$$

$\mathbf{J}_{p}$ is the current density in the plasma,while $\mathbf{J}_{c}$ is the current density in the conductor. We now define the fourier transform $f(\mathbf{k}, \omega)$ of the function $f(\mathbf{x}, t)$ by

$$
f(\mathbf{k}, \omega)=f\{f(\mathbf{x}, t)\}=\frac{1}{(2 p i)^{2}} \int f(\mathbf{x}, t) e^{i(\mathbf{k} \cdot \mathbf{x}-\omega t)} d^{3} x d t
$$

$\mathbf{J}_{p}(\mathbf{k}, \omega)$ is related to $\mathbf{E}(\mathbf{k}, \omega)$ through the dielectric tensor $\mathbf{K}$ by the relation

$$
\mathbf{J}_{p}(\mathbf{k}, \omega)=\frac{i \omega}{4 \pi}[\mathbf{K}(\mathbf{k}, \omega)-\mathbf{I}] \cdot \mathbf{E}(\mathbf{k}, \omega)
$$

where $\mathbf{I}$ is the identity matrix, and $\mathbf{K}$ is the dielectric tensor of the plasma. Although the 
computations that we quote at the end of this paper were performed using the cold plasma dielectric tensor, which is derived from the linearized equztions of motion, any plasma model can be used. Using (9) through (14), we can write the Fourier transform of (8) as

$$
\mathbf{T}(\mathbf{k}, \omega) \cdot \mathbf{E}(\mathbf{k}, \omega)=-\frac{4 \pi i \omega}{c^{2}} \mathbf{J}_{s}(\mathbf{k}, \omega)
$$

where $\mathbf{T}$ is the so-called dispersion tensor, defined by

$$
\mathbf{T}=-k^{2} \mathbf{I}+\mathbf{k} \mathbf{k}+\frac{\omega^{2}}{c^{2}} \mathbf{K}
$$

Eq. (16) can be inverted to solve for $\mathbf{E}$ in terms of $\mathbf{J}_{s}$

$$
\mathbf{E}(\mathbf{k}, \omega)=-\frac{4 \pi i \omega}{c^{2}} \mathbf{T}^{-1}(\mathbf{k}, \omega) \cdot \mathbf{J}_{s}(\mathbf{k}, \omega)
$$

Once $\mathbf{E}(\mathbf{k}, \omega)$ is known, the magnetic field perturbation $\mathbf{B}$, the plasma current density $\mathbf{J}_{p}$, and the electric charge density $\rho$ can easily be found from the Fourier transforms of Maxwell's equations. We seek an equation for $\mathbf{J}_{s}$. To derive it, we multiply Eq. (6) by $H(\mathbf{x}, t)$ and use Eq. (11) to eliminate $\mathbf{J}_{c}$. The result is in the linlearized approximation $\mathbf{V} \times \mathbf{B} \approx \mathbf{V} \times \mathbf{B}_{0}$ is

$$
\mathbf{J}_{s}(\mathbf{x}, t)=\left[\sigma\left[\mathbf{E}(\mathbf{x}, t)+\frac{\mathbf{V} \times \mathbf{B}_{0}}{c}\right]-\mathbf{J}_{p}(\mathbf{x}, t)+\rho_{c}(\mathbf{x}, t) \mathbf{V}\right] H(\mathbf{x}, t)
$$

where we have linearized by writing $\mathbf{B}_{0}$ instead of $\mathbf{B}$. We note that the Fourier transforms of the fields on the right side of Eq. (18) can be written in terms of the Fourier transform of $\mathbf{J}_{s}$. The result is the desired integral equation for $\mathbf{J}_{S}$.

$$
\begin{gathered}
f^{-1}\left\{\mathbf{J}_{s}(\mathbf{k}, \omega)\right\}=0 \quad \text { for } \mathbf{x} \text { in the plasma } \\
f^{-1}\left\{\mathbf{W} \cdot \mathbf{J}_{s}\right\}=\frac{\mathbf{V} \times \mathbf{B}_{0}}{c} \quad \text { for } \mathbf{x} \text { in the conductor }
\end{gathered}
$$

where the tensor $\mathbf{W}$ is defined by

$$
\mathbf{W}=\frac{4 \pi i \omega}{c^{2}} \mathbf{T}^{-1}+\frac{1}{\sigma}\left[\left(\mathbf{k}^{2}-\frac{\omega^{2}}{c^{2}}\right) \mathbf{I}-\mathbf{k k}\right] \cdot \mathbf{T}^{-1}
$$

We are led to an integral equation by the facts that the relation between $\mathbf{E}$ and $\mathbf{J}_{s}$ is an algebraic relation in $(\mathbf{k}, \omega)$ space, while the location of the conductor, as expressed by $H(\mathbf{x}, t)$, is simple in ordinary $(\mathbf{x}, t)$ space. Notice that the boundary conditions are automatically included in our formulation.

\section{Properties of the solutions}

The solution to (19) and (20) contains a complete description of the fields surrounding a conductor moving through a magnetized plasma. Unfortunately, this integral equation is difficult to solve. To obtain numerical estimates, we therefore take the alternate approach of trying to study the properties of the solutions. If we assume that we know the source current 
$\mathbf{J}_{S}$, the electric field is given by the Fourier transform of (17). When one uses a complex contour integration to evaluate the integral, the only contributions come from the poles of the integrand, which occur at the zeros of the equation

$$
\operatorname{det} \mathbf{T}^{-1}=0
$$

Equation (21) is the familiar dispersion relation for the plasma. In the present case, we seek a solution which is independent of time in $\Sigma^{\prime}$, which implies that in $\Sigma$,

$$
\omega=\mathbf{k} \cdot \mathbf{V}
$$

For the geometry described in (1) - (4), (22) takes the form

$$
\omega=k_{x} V
$$

Furthermore, since we are primarily interested in the power radiated, we need to study the behavior of the solutions far from the origin. In particular, we are interested only in those modes for which $\mathbf{k}$ and $\omega$ are both real, since only these modes can transport energy to infinity. In low Earth equatorial orbit, the plasma and the orbital velocity obey the following inequalities

$$
\omega_{p}^{2} \gg \Omega_{e}^{2}
$$

and

$$
V^{2} \ll c_{A}^{2} \ll c^{2}
$$

For such conditions, (21) and (23) have solutions for real $\mathbf{k}$ and $\omega$ in only three frequency bands

$$
\begin{array}{ll}
\omega<\Omega_{i} & \text { Band I } \\
\omega_{! h}<\omega<\Omega_{\varepsilon} & \text { Band II } \\
\omega_{p}<\omega<\omega_{u h} & \text { Band III }
\end{array}
$$

Band I is the MHD band discussed by Drell et al; bands II and III are new. This radiation is analogous to classical Cherenkov radiation. We have already pointed out that a conductor moving through a magnetized plasma only radiates plasma waves whose phase speed in some direction is slower than the speed of the body. For a body that moves slowly compared to the Alfvén speed, this occurs only in the frequency bands described by (26). A polar plot of the phase speed versus the angle between $\mathbf{k}$ and $\mathbf{B}_{0}$ for the mode that is excited in each of these three bands is shown in Figs. 7-9. Note that the phase velocity vanishes in some direction for each of these three cases.

\section{Calculation of radiated power}

Having identified the frequency bands within which power is radiated, we now have to estimate the amount of power that will be radiated into each band. To do this, we guess the spatial dependence of the source current distribution and use Poynting's theorem to compute its magnitude. Poynting's theorem can be written as follows

$$
P_{\text {rad }}=-W-\frac{\partial U_{E M}}{\partial t}
$$


where

$$
\begin{gathered}
P_{\text {rad }}=\frac{c}{4 \pi} \int \mathbf{E} \times \mathbf{B} \cdot d \mathbf{a} \\
W=\int \mathbf{E} \cdot \mathbf{J} d \mathbf{x} \\
U_{E M}=\frac{1}{8 \pi} \int\left(E^{2}+B^{2}\right) d \mathbf{x}
\end{gathered}
$$

where $P_{\text {rad }}$ is the power radiated, $U_{E M}$ is the electro-magnetic energy, and $W$ is the mechanical work done by the fields. We choose for our volume of integration a large rectangular prism with the conductor at its center, and we perform all of the calculations in frame $\Sigma$. If we make the box large enough, the term $\frac{\partial U_{E M}}{\partial t}$ can be neglected, due to the fact that the solution we seek is independent of time in frame $\Sigma^{\prime}$. To evaluate the remaining integrals, we use the following ansatz; assume that the source current can be written as

$$
\mathbf{J}_{s}(\mathbf{x})=-I f(\mathbf{x}) \mathbf{e}_{y}
$$

where $f(\mathbf{x})$ is a known function, the form of which one guesses from the geometry of the conductor. Now consider (28). Since both $\mathbf{E}$ and $\mathbf{B}$ are linear functions of $\mathbf{J}_{s}, P_{\text {rad }}$ is proportional to $I^{2}$. To evaluate (29), we use Ohm's law (6) for $\mathbf{E}$. Since $\mathbf{J}_{c}$ is proportional to $\mathbf{J}_{s}$, (29) results in two terms, one proportional to $I$ and one proportional to $I^{2}$. We can therefore write (27)-(29) as

$$
I^{2} Z_{\text {rad }}=I\left(\phi-I^{2} R\right)
$$

where $\phi$ has dimensions of electric potential, and we have assumed that $\mathbf{J}_{s} \approx \mathbf{J}_{c} . Z_{\text {rad }}$, $R$, and $\phi$ can be computed using $(31),(17),(13),(28)$ and (29). Equation (32) can be interpreted as an electrical circuit analog, where $\phi$ is the voltage, $I$ is the current, and $Z_{\text {rad }}$ and $R$ are the resistences of two resistors in series. The problem of computing the radiated power thus reduces to evaluating $Z_{\text {rad }}$, given by

$$
Z_{r a d}=\frac{c}{4 \pi I^{2}} \int(\mathbf{E} \times \mathbf{B}) \cdot d \mathbf{a}
$$

with $\mathbf{E}$ and $\mathbf{B}$ given by

$$
\mathbf{E}(\mathbf{x}, t)=f^{-1}\left\{-\frac{4 \pi i \omega}{c^{2}} \mathbf{T}^{-1} \cdot f(\mathbf{k}) \mathbf{e}_{x}\right\} I
$$

and

$$
\mathbf{B}=f^{-1}\left\{\frac{c}{\omega} \mathbf{k} \times \mathbf{E}\right\}
$$

where $f(\mathbf{k})$ is the Fourier transform of $f(\mathbf{x})$ defined in (31). We have evaluated (33) explicitly for two different geometries. The first case is a sphere of radius $a$. The second is a cylinder of radius $b$ and length $L$ whose axis points in the $\mathbf{e}_{y}$ direction. In both cases we have assumed a current density of the form given in (31), with $f(\mathbf{x})$ a constant inside the object and zero outside it. In both cases $Z_{\text {rad }}$ can be expressed as a sum of three terms 


$$
Z_{\text {rad }}=Z_{I}+Z_{I I}+Z_{I I I}
$$

where $Z_{I}, Z_{I I}$, and $Z_{I I}$ are the contributions from bands I, II, and III, respectively. Analytic expressions for $Z$ as a function of $a$ and of $b$ and $L$ can be found for several limiting cases. These cases are summarized in Table 1 . The results of numerical calculations of $Z_{I}, Z_{I I}, Z_{I I}$, and $P_{r a d}$ are shown in Figs. 10-13, and the plasma parameters used in the calculations are given in Table 2. Of considerable interest are the results for a long, thin wire, since this might have applications to tethered satellite systems. If one wants to estimate the power that can be generated using a TSS, one can simply consider $R$ to be the load resistence. Due to the contributions from band $\Pi$, the impedence is much higher than expected in this case. A cylinder with $L=10 \mathrm{~km}$ and $b=1 \mathrm{~cm}$ has a radiation impedence of nearly $10^{5}$ ohms! These results suggest that previous power estimates for passive TSS systems might be much too high. One must use care when applying these results to the TSS, however. In particular, effects due to the size of the subsatellite and local plasma clouds from "plasma contactors" can be mocked up by using an "effective" b.

Our formulation can also be used to estimate the radiated power for active systems. One must then consider $I$ in (31) as a known source strength Some calculations of that sort are discussed in the article by Hastings et al.

\section{Summary}

We have considered the problem of the interaction between a moving conductor and a magnetized plasma. We have shown that steady state solutions exist for which the body is surrounded by a system of standing waves. Wave modes are excited for which the phase speed in some direction is less than the speed of the conductor. The process is analogous to Cherenkov radiation, or to the formation of a shock wave surrounding a body in supersonic motion through a gas. We have extimated the powcr radiated for spherical and cylindrical bodies moving perpendicular to the background magnetic field. Our results suggest that, due to the high radiation impedence in band $\Pi$, a passive electrodynamic tethered satellite system will not draw large currents.

Acknowledgements We wish to thank Dr. Zdzislaw Musielak for his work on the polar plots of the plasma wave phase velocities. This work was supported in part by AFGL contract 719628-86-k-0027.

\section{References}

Acuna, M. H.,and F. M. Ness, Standing Alfvén wave current system at Io: Voyager I observations, J. Geophys. Res., 86, 8513, 1981

Barnett,A., In situ measurements of the bulk plasma velocity near the Io flux tube, J. Geophys. Res., 86, 8513, 1981

Drell, S. D.,H. M. Foley, and M. A. Ruderman, Drag and propulsion in the ionosphere: An Alfvén engine in space, J. Geophys. Res.., 70, $3131,1965$. 
TABLE 1. Radiation Impedence of Conductors Moving Through a Plasma Perpendicular to the Magnetic Field

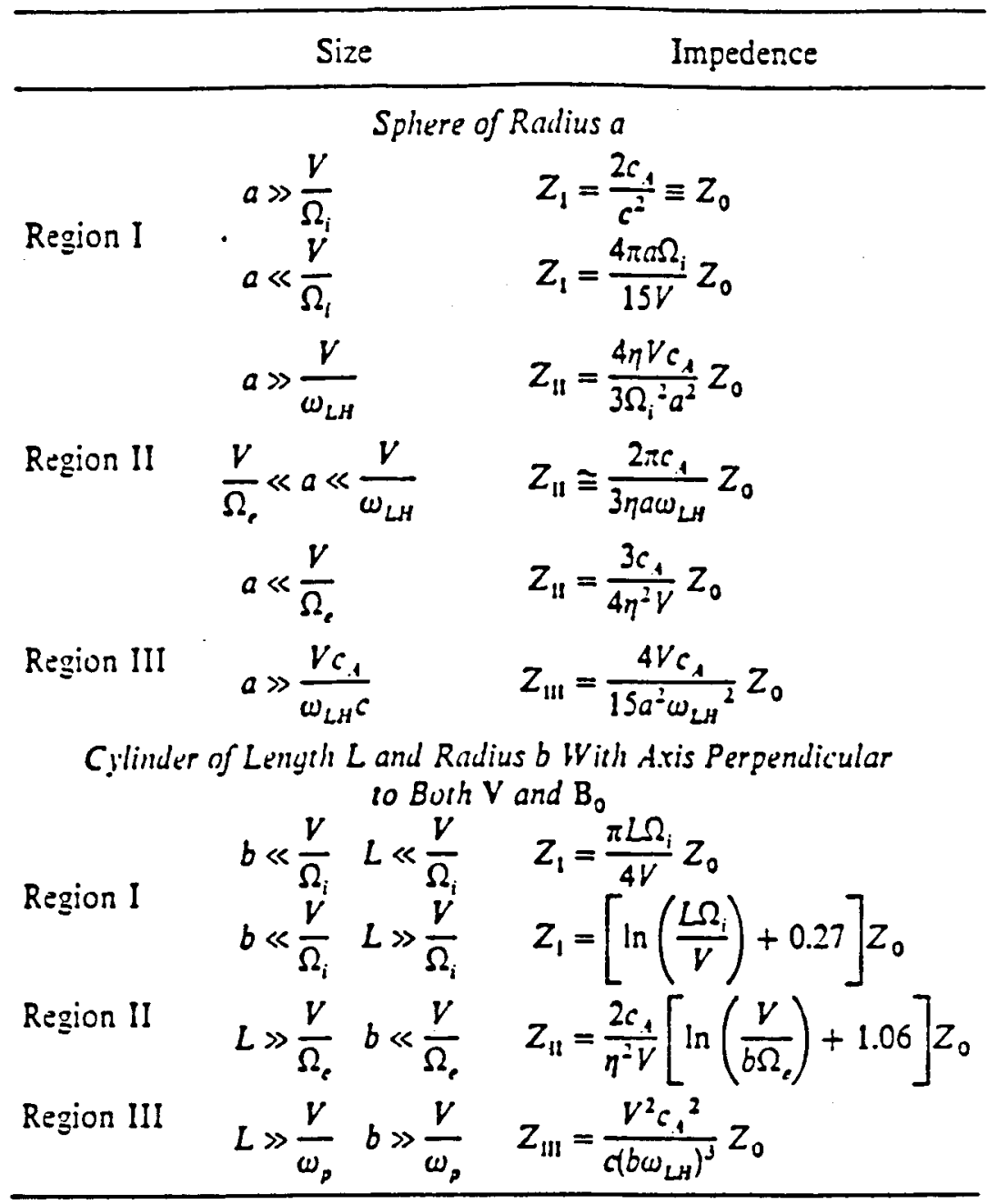

We have expressed the impedence in terms of $Z_{0} \equiv 2 c_{1} / c^{2}$ in Gaussian units. For conditions typical of low earth orbit, $Z_{0}=6.7$ $\times 10^{-1+} s^{\prime} / \mathrm{cm}=0.06 \Omega$ If $\eta=\left(m_{e}^{\prime} m_{j}\right)^{1 / 2}=1 / 172$ (oxygen plasma). $c_{A}=300 \mathrm{~km} / \mathrm{s}$ and $B_{0}=0.33 \mathrm{G}$.

TABLE 2. P!asma Parameters Used in. Numerical Calculations

\begin{tabular}{ll}
\hline Parameter & \multicolumn{1}{c}{ Value } \\
\hline$B$ & $0.33 \mathrm{G}$ \\
$n_{e}$ & $3.6 \times 10^{9} \mathrm{~cm}^{-3}$ \\
$\eta$ & $1 / 172$ \\
$V_{1}$ & $7.3 \mathrm{~km} / \mathrm{s}$ \\
$C_{A}$ & $300 \mathrm{~km} / \mathrm{s}$ \\
$H_{A}$ & 0.024 \\
$\omega_{,}$ & $3.4 \times 10^{2} \mathrm{~s}^{-1}$ \\
$\Omega_{e}$ & $5.9 \times 10^{6} \mathrm{~s}^{-1}$ \\
$\omega_{L H}$ & $3.5 \times 10^{4} \mathrm{~s}^{-1}$ \\
$\Omega_{i}$ & $2.0 \times 10^{2} \mathrm{~s}^{-1}$ \\
$l / k_{e}$ & $1.2 \mathrm{~mm}$ \\
$1 / k_{L H}$ & $21 \mathrm{~cm}$ \\
$1 / k_{i}$ & $36 \mathrm{~m}$ \\
\hline
\end{tabular}




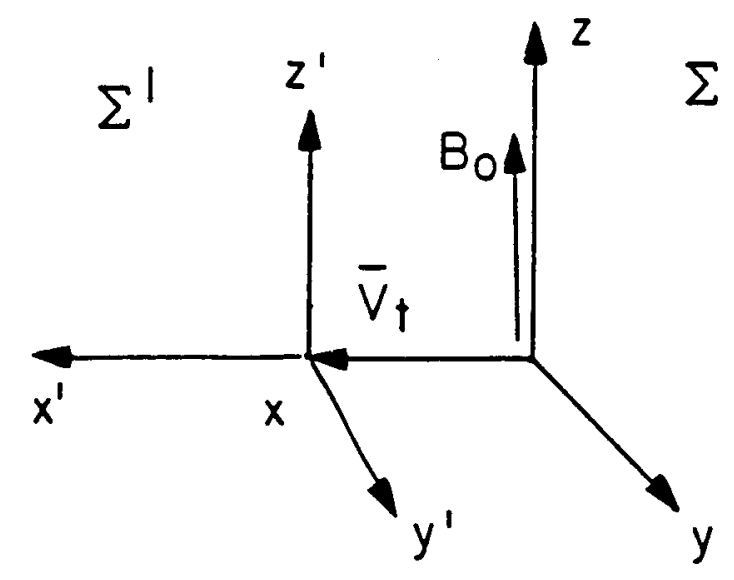

Fig. 1. Coordinate System

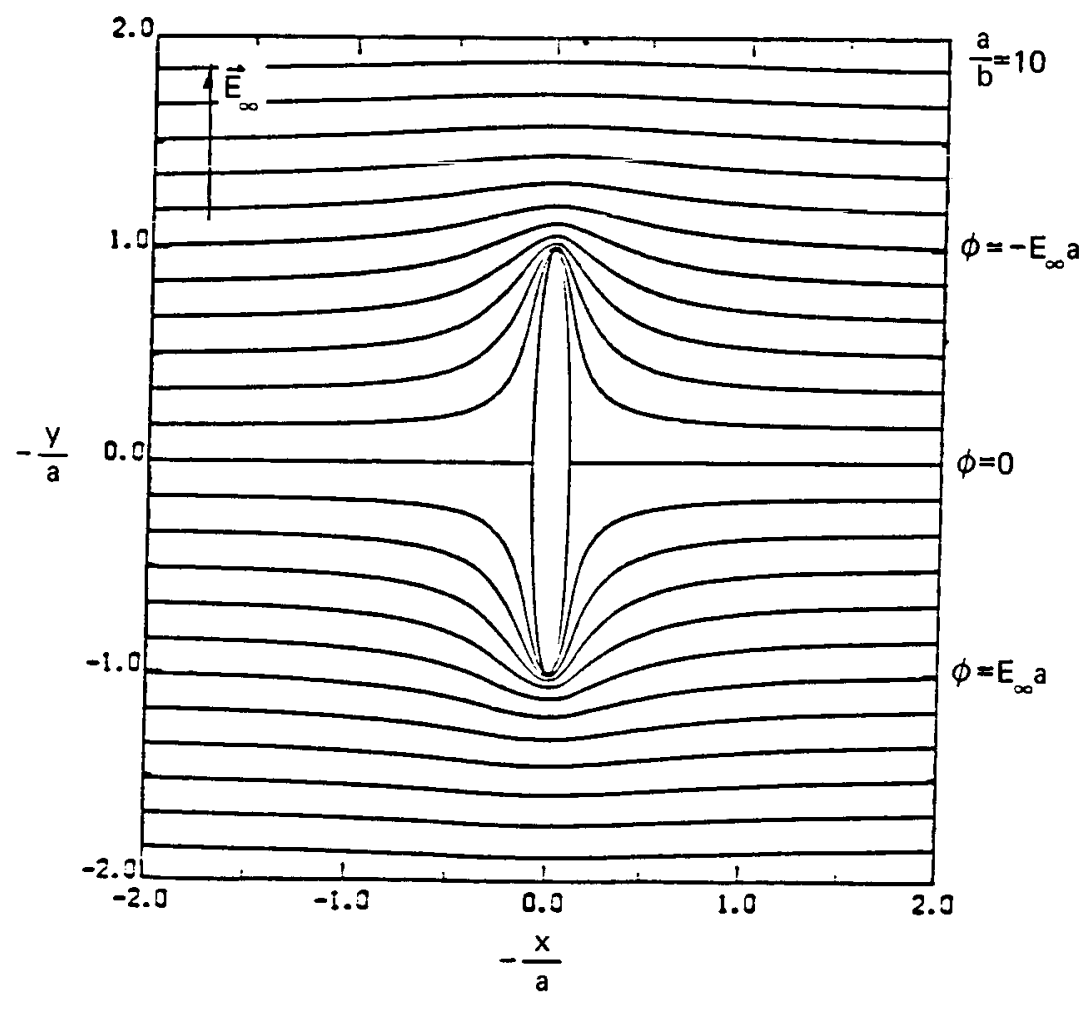

Fig. 2. Equipotential surfaces

for prolate spheroid 

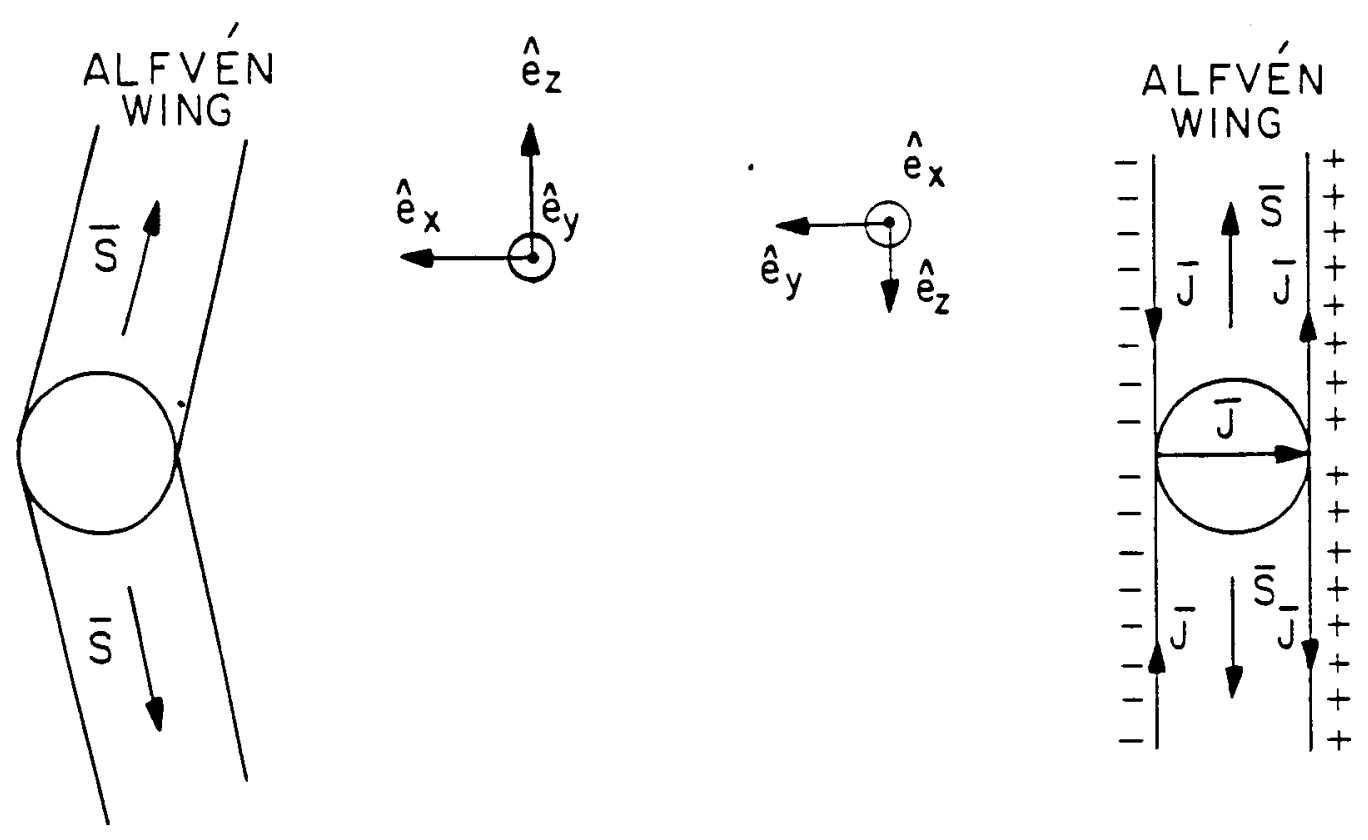

Fig. 4. Alfven wing for perfectly conducting sphere-front view

Fig. 3. Alfven wing for perfectly conducting sphere-side view
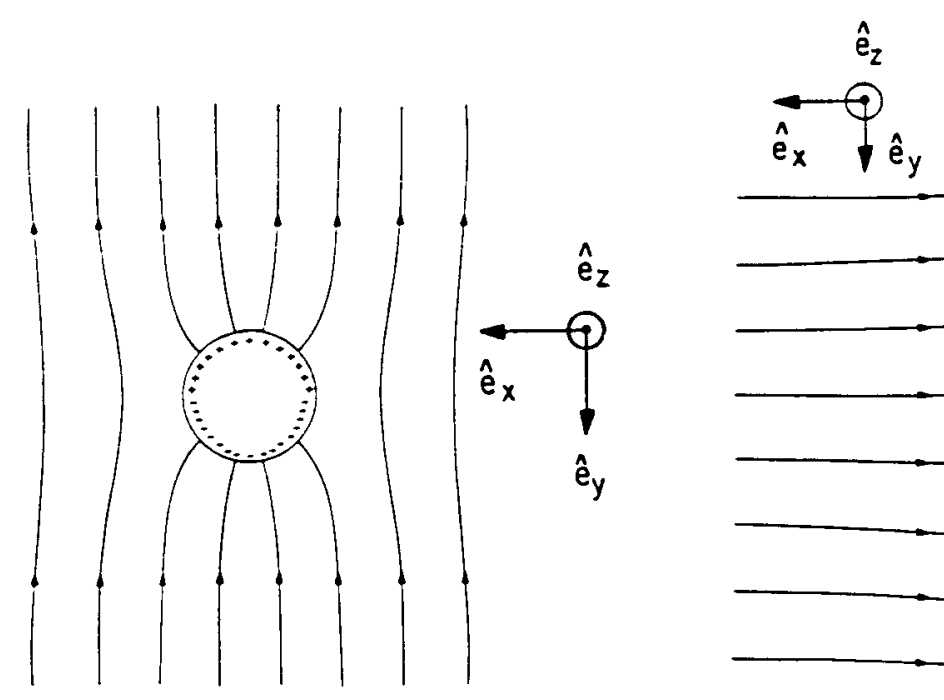

Fig. 5. Electric field lines in equatorial piane for perfectly conducting sphere

Fig. 6. Stream lines in equatorial plane for flow around perfectiy conducting sphere 


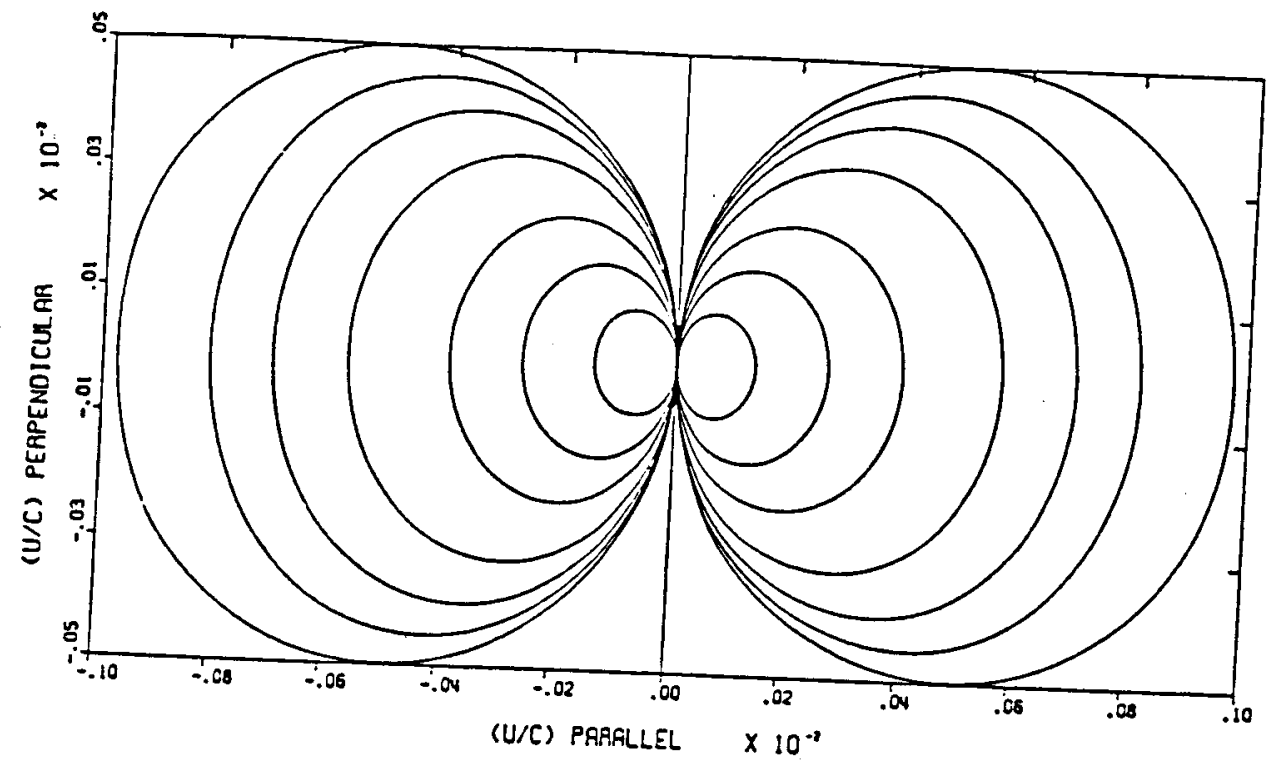

Fig. 7. Phase velocity vs. angle for 7 different
frequencies in Band I
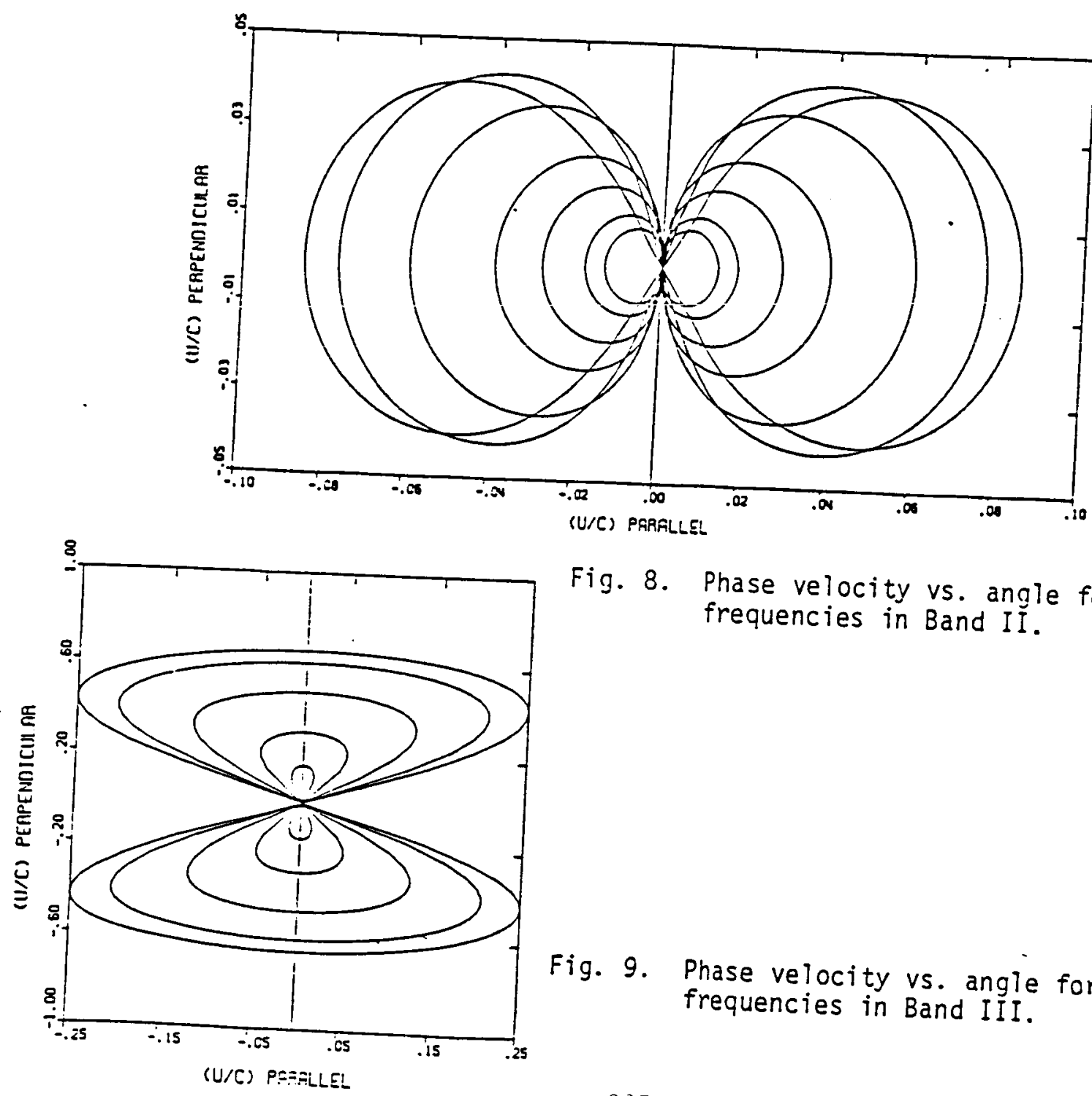

Fig. 8. Phase velocity vs. angle for 7 different
frequencies in Band II.

Fig. 9. Phase velocity vs. angle for 5 different
frequencies in 


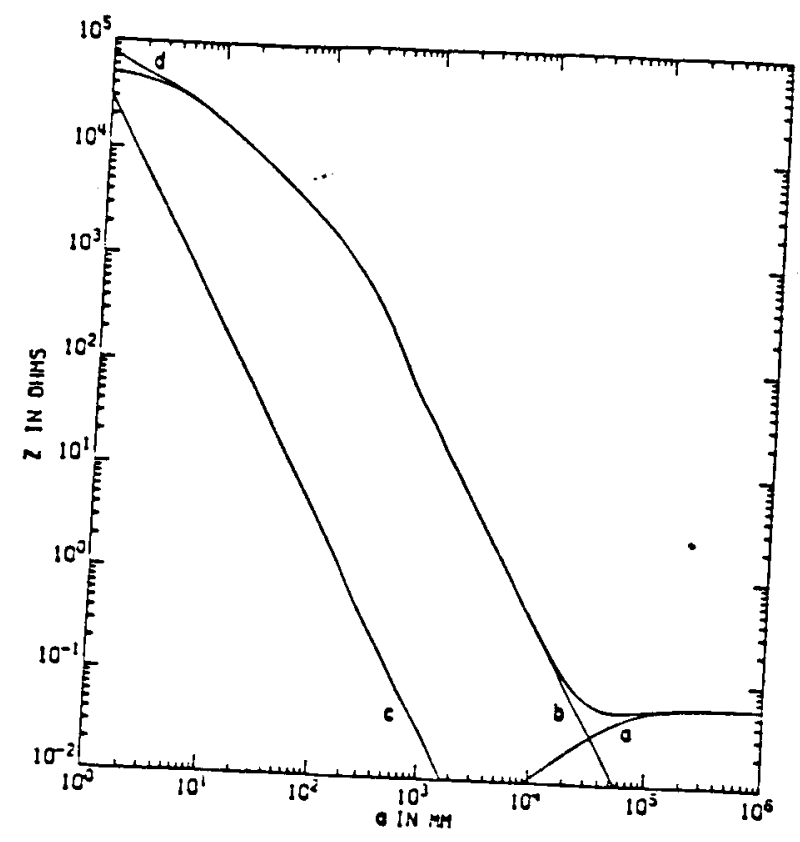

Fig. 10. Impedence vs, radius for a sphere. The lines labelled $a, b$, $c$, and $d$ are the contributions from bands I, II, III, and total, respectively. Plasma parameters are given
in Table 2 .

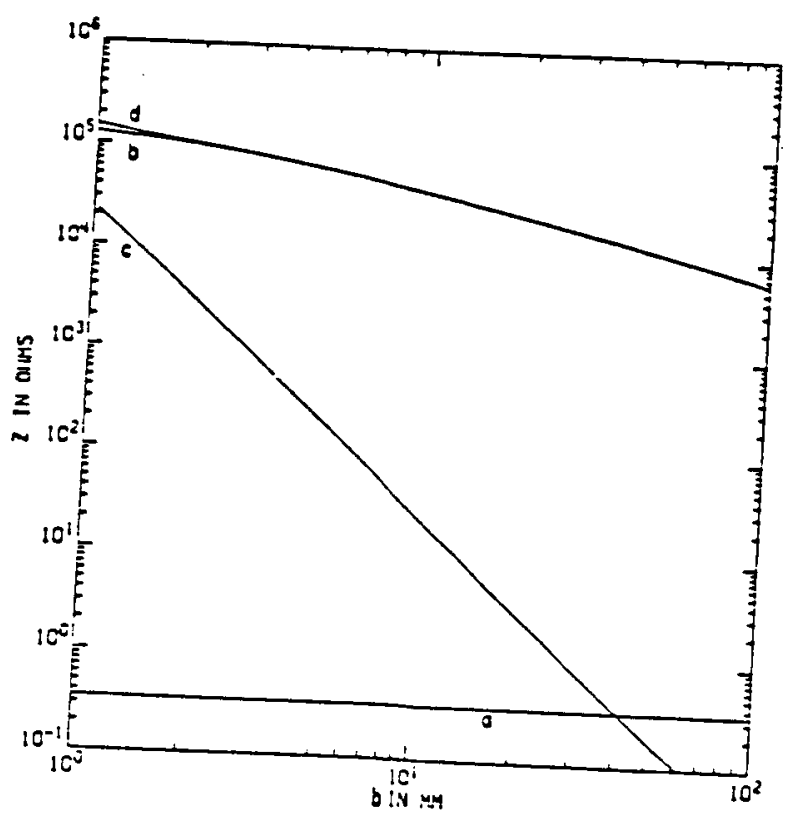

Fig. 12. Impedence vs, radius for a $10 \mathrm{~km}$ long cyl inder. The lines labelled $a, b, c$, and $d$ are the contributions from bands I, II, III and total, respectively. Plasma parameters are given in Table 2 .

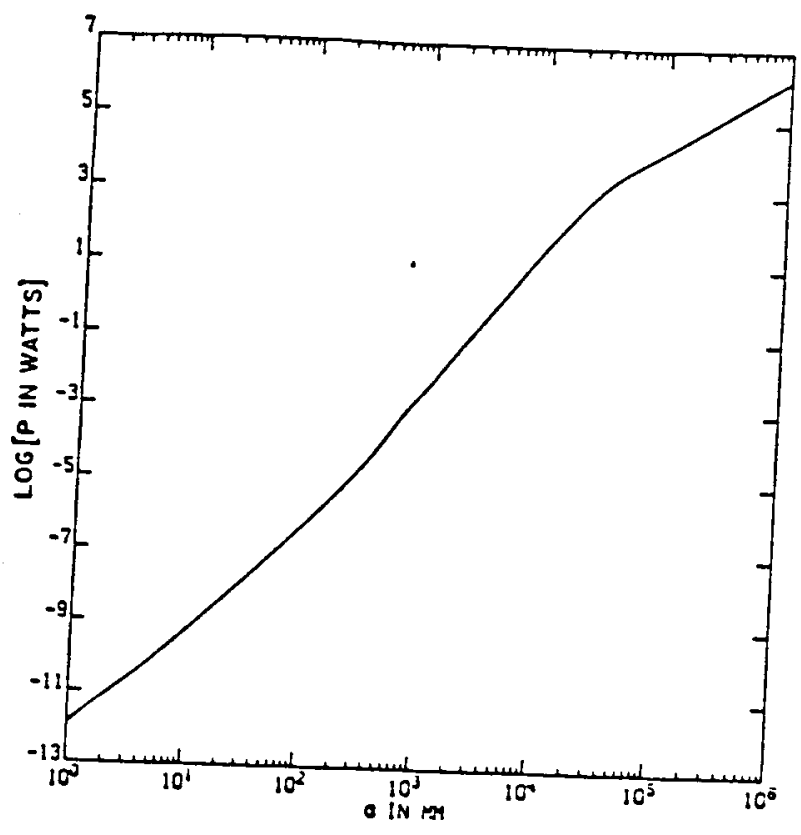

Fig. 11. Power radiated vs. radius for a sphere. Plasma parameters used are given in Table 2 .

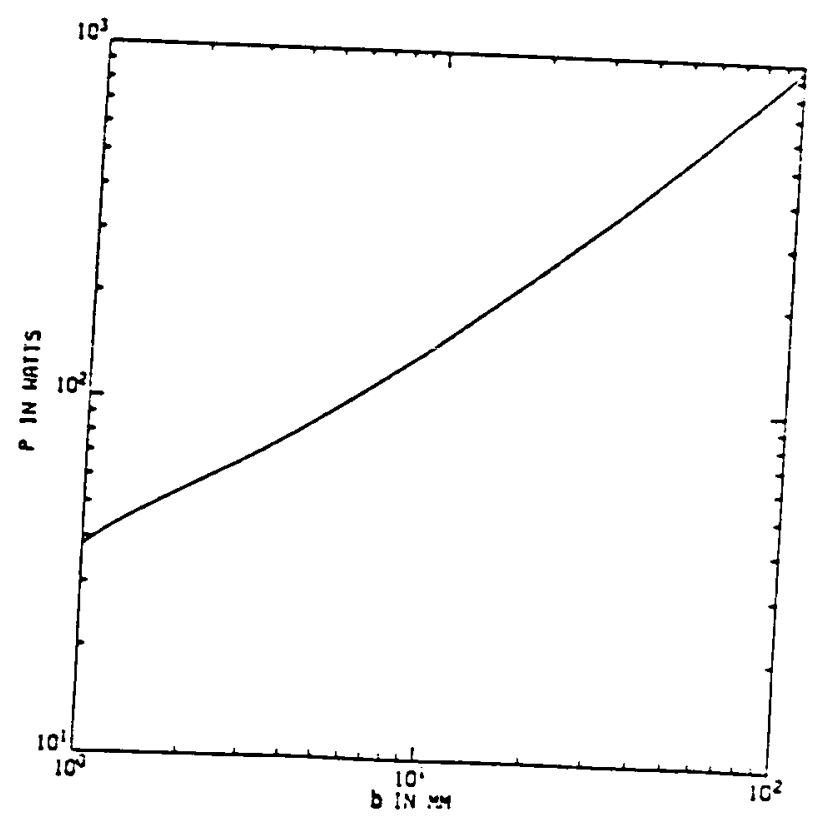

Fig. 13 . Power radiated vs. radius for a $10 \mathrm{~km}$ long cylinder. plasma parameters used are given in Tabie 2 . 